\title{
Solving Disjunctive/Conjunctive Boolean Equation Systems with Alternating Fixed Points
}

\author{
Jan Friso Groote ${ }^{2,3}$ and Misa Keinänen ${ }^{1,3}$
}

1 Dept. of Computer Science and Engineering, Lab. for Theoretical Comp. Science

Helsinki University of Technology, P.O. Box 5400, FIN-02015 HUT, Finland

Misa.Keinanen@hut.fi

2 Departement of Mathematics and Computer Science, Eindhoven University of Technology, P.O. Box 513, 5600 MB Eindhoven, The Netherlands

3 CWI, P.O. Box 94079, 1090 GB Amsterdam, The Netherlands J.F.Groote@tue.nl,

\begin{abstract}
This paper presents a technique for the resolution of alternating disjunctive/conjunctive boolean equation systems. The technique can be used to solve various verification problems on finite-state concurrent systems, by encoding the problems as boolean equation systems and determining their local solutions. The main contribution of this paper is that a recent resolution technique from [13] for disjunctive/conjunctive boolean equation systems is extended to the more general disjunctive/conjunctive forms with alternation. Our technique has the time complexity $O\left(m+n^{2}\right)$, where $m$ is the number of alternation free variables occurring in the equation system and $n$ the number of alternating variables. We found that many $\mu$-calculus formulas with alternating fixed points occurring in the literature can be encoded as boolean equation systems of disjunctive/conjunctive forms. Practical experiments show that we can verify alternating formulas on state spaces that are orders of magnitudes larger than reported up till now.
\end{abstract}

\section{Introduction}

Modal $\mu$-calculus [10] is an expressive logic for system verification, and most model checking logics can be encoded in the $\mu$-calculus. Many important features of system models, like equivalence/preorder relations and fairness constraints, can be expressed with the logic, also. For these reasons, $\mu$-calculus is a logic widely studied in the recent systems verification literature.

It is well-known that the $\mu$-calculus model checking problem is in the complexity class NP $\cap$ co-NP. Emerson, Jutla, and Sistla [78] showed the problem can be reduced to determining the winner in a parity game, and thus is in NP (and also by symmetry in co-NP). More recently, Jurdzinsky [9] showed that the problem is even in $\mathrm{UP} \cap$ co-UP. Yet the complexity of $\mu$-calculus model checking problem for the unrestricted logic is an open problem; no polynomial algorithm has been discovered.

Nevertheless, various effective model checking algorithms exist for expressive subsets. Arnold and Crubille 2] presented an algorithm for checking alternation depth 1 formulas of $\mu$-calculus, which is linear in the size of the model and 
quadratic in the size of the formula. Cleaveland and Steffen [6] improved the result by making the algorithm linear also in the size of the formula. Andersen [1, and similarly Vergauwen and Lewi [16], showed how model checking alternation depth 1 formulas amounts to the evaluation of boolean graphs, resulting also in linear time techniques for model checking alternation depth 1 formulas. Even more expressive subsets of $\mu$-calculus were investigated by Bhat and Cleaveland 5 as well as Emerson et al. 78. They presented polynomial time model checking algorithms for fragments L1 and L2, which may contain alternating fixed point formulas.

In this paper, instead of treating $\mu$-calculus expressions together with their semantics, we prefer to work with the more flexible formalism of boolean equation systems [121317]. Boolean equation systems provide a useful framework for studying verification problems of finite-state concurrent systems, because $\mu$ calculus expressions can easily be translated into this simple formalism (see e.g. 31213 for such translations).

We restrict the attention to boolean equation systems, which are either in disjunctive or in conjunctive form. We found that many practically relevant $\mu$ calculus formulas (actually virtually all of them) can be encoded as boolean equation systems that are disjunctive, conjunctive, or disjunctive/conjunctive straight (see definition 3). For instance, the model checking problems for Hennessy-Milner logic (HML), Computation Tree Logic (CTL), and many equivalence/preorder checking problems result in alternation-free boolean equation systems in disjunctive/conjunctive forms (see for instance [13]). Moreover, encoding the L1 and L2 fragments of the $\mu$-calculus (and similar subsets) or many fairness constraints as boolean equation systems result in alternating systems which are in disjunctive/conjunctive form.

Hence, the problem of solving disjunctive/conjunctive boolean equation systems with alternating fixed points is so important that developing special purpose solution techniques for these classes is worthwhile. Recently, the question has been addressed by Mateescu [13], who presented a resolution algorithm for disjunctive/conjunctive boolean equation systems. But, this approach is restricted to alternation-free systems. We are only aware of one sketch of an algorithm that is directed to alternating disjunctive/conjunctive boolean equation systems (proposition 6.5 and 6.6 of [12]). Here a $O\left(n^{3}\right)$ time and $O\left(n^{2}\right)$ space algorithm is provided where $n$ is the number of variables 1 . Our algorithm is a substantial improvement over this.

In this paper, we address the problem of solving alternating disjunctive/conjunctive straight boolean equation systems. The algorithm for the resolution of such equation systems is quite straightforward comparable to the alternationfree case presented in 13 . Essentially, the idea consists of computing simple kinds of dependencies between certain variables occurring in the equation sys-

\footnotetext{
${ }^{1}$ The paper 12 claims an $O\left(n^{2}\right)$ time algorithm, assuming the existence of an algorithm which allows union of (large) sets, and finding and deletion of elements in these in constant time. To our knowledge for this only a linear and most certainly no constant time algorithm exists.
} 
tems. Our technique is such that it ensures linear-time worst case complexity of solving alternation-free boolean equation systems, and quadratic for the alternating systems. More precisely, we present resolution algorithms for the disjunctive/conjunctive classes which are of complexity $O\left(m+n^{2}\right)$, where $m$ is the number of alternation-free variables and $n$ the number of alternating variables occurring in the system. Hence, our approach preserves the best known worst case time complexity of model checking of many restricted but expressive fragments of the $\mu$-calculus.

The paper is organized as follows. Section 2 introduces basic notions concerning boolean equation systems. Section 3 introduces the subclasses of disjunctive, conjunctive and disjunctive/conjunctive straight boolean equation systems and illustrates that many formulas with alternating fixed points fall into these classes. Section 4 presents the algorithm and section 5 provides some initial experimental results. In section 6 we wrap up and provide an open problem that we were unable to solve, but which - if solved - would eliminate the quadratic factor in the time complexity of our algorithm.

\section{Boolean Equation Systems}

We give here a short introduction into boolean equation systems. A boolean equation system is an ordered sequence of fixed point equations like

$$
\left(\sigma_{1} x_{1}=\alpha_{1}\right)\left(\sigma_{2} x_{2}=\alpha_{2}\right) \ldots\left(\sigma_{n} x_{n}=\alpha_{n}\right)
$$

where all $x_{i}$ are different. We generally use the letter $\mathcal{E}$ to represent a boolean equation system, and let $\epsilon$ stand for the empty boolean equation system. The symbol $\sigma_{i}$ specifies the polarity of the fixed points. The symbol $\sigma_{i}$ is $\mu$ if the $i$-th equation is a least fixed point equation and $\nu$ if it is a greatest fixed point equation. The order of equations in a boolean equation system is very important, and we keep the order on variables and their indices in strict synchrony. We write $\mathcal{X}=\left\{x_{1}, x_{2}, \ldots, x_{n}\right\}$ for the set of all boolean variables. For each $1 \leq i \leq n$ we allow $\alpha_{i}$ to be a formula over boolean variables and constants false and true and operators $\wedge$ and $\vee$, summarized by the grammar:

$$
\alpha::=\text { true } \mid \text { false }|x \in \mathcal{X}| \alpha_{1} \wedge \alpha_{2} \mid \alpha_{1} \vee \alpha_{2} .
$$

We write $x_{i} \in \alpha_{j}$ if $x_{i}$ is a subterm of $\alpha_{j}$.

The semantics of boolean equation systems provides a uniquely determined solution, to each boolean equation system $\mathcal{E}$. A solution is a valuation assigning a constant value in $\{0,1\}$ (with 0 standing for false and 1 for true) to all variables occurring in $\mathcal{E}$. Let $v, v_{1}, \ldots$ range over valuations, where each $v$ is a function $v: \mathcal{X} \rightarrow\{0,1\}$. We extend the definition of valuations to terms in the standard way. So, $v(\alpha)$ is the value of the term $\alpha$ after substituting each free variable $x$ in $\alpha$ by $v(x)$. Let $v[x:=a]$ denote the valuation that coincides with $v$ for all variables except $x$, which has the value $a$. We suppose that $[x:=a]$ has priority over all operations and $v[x:=a]$ stands for $(v[x:=a])$. Similarly, we apply $[x:=a]$ to terms; 
$\alpha[x:=a]$ indicates the term $\alpha$ where all occurrences of $x$ have been replaced by $a$.

Definition 1 (The solution of a boolean equation system). The solution of a boolean equation system $\mathcal{E}$ relative to a valuation $v$, denoted by $\llbracket \mathcal{E} \rrbracket v$, is an assignment inductively defined by

$$
\begin{aligned}
& \llbracket \epsilon \rrbracket v=v \\
& \llbracket\left(\sigma_{i} x_{i}=\alpha_{i}\right) \mathcal{E} \rrbracket v=\left\{\begin{array}{l}
\llbracket \mathcal{E} \rrbracket v\left[x_{i}:=\mu x_{i} \cdot \alpha_{i}(\llbracket \mathcal{E} \rrbracket v)\right] \text { if } \sigma_{i}=\mu \\
\llbracket \mathcal{E} \rrbracket v\left[x_{i}:=\nu x_{i} \cdot \alpha_{i}(\llbracket \mathcal{E} \rrbracket v)\right] \text { if } \sigma_{i}=\nu
\end{array}\right.
\end{aligned}
$$

where $\mu x_{i} \cdot \alpha(\llbracket \mathcal{E} \rrbracket v)=\bigwedge\left\{a \mid \alpha_{i}(\llbracket \mathcal{E} \rrbracket v[x:=a]) \Rightarrow a\right\}$ and $\nu x_{i} \cdot \alpha(\llbracket \mathcal{E} \rrbracket v)=\bigvee\{a \mid a \Rightarrow$ $\left.\alpha_{i}(\llbracket \mathcal{E} \rrbracket v[x:=a])\right\}$.

It is said that a variable $x_{i}$ depends on variable $x_{j}$, if $\alpha_{i}$ contains a reference to $x_{j}$, or to a variable $x_{k}$ such that $x_{k}$ depends on $x_{j}$. Two variables $x_{i}$ and $x_{j}$ are mutually dependent if $x_{i}$ depends on $x_{j}$ and vice versa.

A boolean equation system $\mathcal{E}$ is alternation free if, for any two variables $x_{i}$ and $x_{j}$ occurring in $\mathcal{E}, x_{i}$ and $x_{j}$ are mutually dependent implies $\sigma_{i}=\sigma_{j}$. Otherwise, system $\mathcal{E}$ is said to be alternating and it contains alternating fixed points.

Example 1. Let $\mathcal{X}$ be the set $\left\{x_{1}, x_{2}, x_{3}\right\}$ and assume we are given a boolean equation system

$$
\mathcal{E}_{1} \equiv\left(\left(\mu x_{1}=x_{1} \wedge x_{2}\right)\left(\mu x_{2}=x_{1} \vee x_{2}\right)\left(\nu x_{3}=x_{2} \wedge x_{3}\right)\right) .
$$

The system $\mathcal{E}_{1}$ is alternation-free, because it does not contain mutually dependent variables with different signs. Yet, note that variable $x_{3}$ with $\operatorname{sign} \sigma_{3}=\nu$ depends on variables $x_{1}$ and $x_{2}$ with different sign. A solution of $\mathcal{E}_{1}$ is given by the valuation $v: \mathcal{X} \rightarrow\{0,1\}$ defined by $v\left(x_{i}\right)=0$ for $i=1,2,3$.

Example 2. Let $\mathcal{X}$ be the set $\left\{x_{1}, x_{2}, x_{3}\right\}$ and assume we are given a boolean equation system

$$
\mathcal{E}_{2} \equiv\left(\left(\nu x_{1}=x_{2} \wedge x_{1}\right)\left(\mu x_{2}=x_{1} \wedge x_{3}\right)\left(\nu x_{3}=x_{3} \vee \text { true }\right)\right) .
$$

The system $\mathcal{E}_{2}$ is alternating, because it contains mutually dependent variables with different signs, like $x_{1}$ and $x_{2}$ with $\sigma_{1} \neq \sigma_{2}$. A solution of $\mathcal{E}_{2}$ is given by the valuation $v: \mathcal{X} \rightarrow\{0,1\}$ defined by $v\left(x_{i}\right)=1$ for $i=1,2,3$.

In Mader 12] there are two lemmas that allow to solve boolean equation systems. As our proofs are based on these, we restate these here.

Lemma 1 (Lemma 6.2 of [12]). Let $\mathcal{E}_{1}$ and $\mathcal{E}_{2}$ be boolean equation systems and let $\sigma x=\alpha$ and $\sigma x=\alpha^{\prime}$ be boolean equations where

$$
\alpha^{\prime}=\left\{\begin{array}{l}
\alpha[x:=\text { true }] \text { if } \sigma=\nu, \\
\alpha[x:=\text { false }] \text { if } \sigma=\mu .
\end{array}\right.
$$

Then $\llbracket \mathcal{E}_{1}(\sigma x=\alpha) \mathcal{E}_{2} \rrbracket v=\llbracket \mathcal{E}_{1}\left(\sigma x=\alpha^{\prime}\right) \mathcal{E}_{2} \rrbracket v$. 
Lemma 2 (Lemma 6.3 of [12]). Let $\mathcal{E}_{1}, \mathcal{E}_{2}$ and $\mathcal{E}_{3}$ be boolean equation systems and let $\sigma_{1} x_{1}=\alpha, \sigma_{1} x_{1}=\alpha^{\prime}$ and $\sigma_{2} x_{2}=\beta$ be boolean equations where $\alpha^{\prime}=$ $\alpha\left[x_{2}:=\beta\right]$. Then

$$
\llbracket \mathcal{E}_{1}\left(\sigma_{1} x_{1}=\alpha\right) \mathcal{E}_{2}\left(\sigma_{2} x_{2}=\beta\right) \mathcal{E}_{3} \rrbracket v=\llbracket \mathcal{E}_{1}\left(\sigma_{1} x_{1}=\alpha^{\prime}\right) \mathcal{E}_{2}\left(\sigma_{2} x_{2}=\beta\right) \mathcal{E}_{3} \rrbracket v .
$$

\section{Disjunctive/Conjunctive Boolean Equation Systems}

We introduce disjunctive/conjunctive form boolean equation systems in their most elementary form

Definition 2. Let $\sigma x=\alpha$ be a fixed point equation. We call this equation disjunctive if no conjunction symbol $(\wedge)$ appears in $\alpha$, and we call it conjunctive if no disjunction $(\mathrm{V})$ symbol appears in $\alpha$. Let $\mathcal{E}$ be a boolean equation system. We call $\mathcal{E}$ conjunctive (respectively disjunctive) iff each equation in $\mathcal{E}$ is conjunctive (respectively disjunctive).

But our algorithm applies to a much wider class of equation systems, namely those where the conjunction and disjunction symbol are not used in a nested way

Definition 3. Let $\mathcal{E}$ be a boolean equation system. We call $\mathcal{E}$ disjunction/conjunction straight (DCS) iff for all variables $x_{i}$ and $x_{j}$ in $\mathcal{E}$ that are mutually dependent, the equations $\sigma_{i} x_{i}=\alpha_{i}$ and $\sigma_{j} x_{j}=\alpha_{j}$ in $\mathcal{E}$ are both conjunctive or both disjunctive.

Observation $I$. The problem of solving disjunction/conjunctive straight boolean equation systems can be reduced to iteratively dealing with disjuntive or conjuntive boolean equation systems as follows. In a DCS boolean equation system the variables can be partitioned in blocks such that variables that mutually depend on each other belong to the same block. The dependency relation among variables can be extended to blocks in the sense that block $B_{i}$ depends on block $B_{j}$ if some variable $x_{i} \in B_{i}$ depends on some variable $x_{j} \in B_{j}$. This dependency relation is an ordering. We can start to find solutions for the variables in the last block, setting them to true or false. Using lemma 2 we can substitute the solutions for variables in blocks higher up in the ordering.

The following simplification rules can be used to simplify the equations

$-(\phi \wedge$ true $) \mapsto \phi$

$-(\phi \wedge$ false $) \mapsto$ false

$-(\phi \vee$ true $) \mapsto$ true

$-(\phi \vee$ false $) \mapsto \phi$

and the resulting equation system has the same solution. The rules allow to remove each occurrence of true and false in the right hand side of equations, except if the right hand side becomes equal to true or false, in which case yet another equation has been solved. By recursively applying these steps all non 
trivial occurrences of true and false can be removed from the equations and we call the resulting equations purely disjunctive or purely conjunctive.

Note that each substitution and simplification step reduces the number of occurrences of variables or the size of a right hand side, and therefore, only a linear number of such reductions are applicable.

After solving all equations in a block, and simplifying subsequent blocks the algorithm can be applied to the blocks higher up in the ordering iteratively solving them all.

Note that this allows us to restrict our attention to algorithms to solve purely disjunctive/conjunctive straight systems.

Example 3. Consider the boolean equation system $\mathcal{E}_{2}$ of example 2 The system $\mathcal{E}_{2}$ is not in conjunctive form. An equivalent conjunctive equation system $\mathcal{E}_{3}$ is obtained by replacing $\alpha_{3}$ of $\mathcal{E}_{2}$ with true and propagating $x_{3}=$ true throughout the formula using lemma 2 This results in the following sequence

$$
\mathcal{E}_{3}=\left(\left(\nu x_{1}=x_{2} \wedge x_{1}\right)\left(\mu x_{2}=x_{1}\right)\left(\nu x_{3}=\text { true }\right)\right)
$$

within which no disjunctions occur in right-hand sides of equations.

Observation II. We found that many formulas with apparently alternating fixed points lead to boolean equation systems that are disjunction/conjunction straight and therefore can be solved efficiently with our techniques.

Consider for instance the examples in section 3.5 in [4. All formulas applied to any labelled transition systems yield disjunction/conjunction straight boolean equation systems, except for the modal formula

$$
\mu Y . v Z .(P \wedge[a] Y) \vee(\neg P \wedge[a] Z) .
$$

But this formula is equivalent to the formula

$$
\mu Y \cdot(([a] Y \vee \nu Z \cdot(\neg P \wedge[a] Z)))
$$

which does lead to DCS equation systems.

As an illustration we explain the transformation of the last formula in the example section of [4]:

$$
\nu X . \mu Y . \nu Z .[a] X \wedge(\langle a\rangle \text { true } \Rightarrow[-a] Y) \wedge[-a] Z .
$$

If we consider a labeled transition system $M=(S, A, \longrightarrow)$ then the boolean equation system looks like:

$$
\left.\begin{array}{l}
\nu x_{s}=y_{s} \\
\mu y_{s}=z_{s} \\
\nu z_{s}=\bigwedge_{s^{\prime} \in \nabla(a, s)} x_{s^{\prime}} \wedge\left(\bigwedge_{s^{\prime} \in \nabla(a, s)} \text { false } \vee \bigwedge_{s^{\prime} \in \nabla(\neg a, s)} y_{s^{\prime}}\right) \wedge \bigwedge_{s^{\prime} \in \nabla(\neg a, s)} z_{s^{\prime}}
\end{array}\right\} \text { for all } s \in S .
$$

Here, $\nabla(a, s):=\left\{s^{\prime} \mid s \stackrel{a}{\longrightarrow} s^{\prime}\right\}$ and $\nabla(\neg a, s):=\left\{s^{\prime} \mid s \stackrel{b}{\longrightarrow} s^{\prime}\right.$ and $\left.b \neq a\right\}$. On first sight these equations do not appear to be a conjunctive boolean equation 
system, as in the third group of equations a disjunction occurs. However, for each concrete labelled transition system the left side of this disjunction will either become true or false for each state $s \in S$. By applying the simplification rules the formula quickly becomes conjunctive.

\section{The Algorithm}

We develop our resolution algorithm in terms of a variable dependency graph similar to those of boolean graphs [1, which provide a representation of the dependencies between variables occurring in equation systems.

Definition 4 (Variable dependency graph). Let $\mathcal{E}=\left(\left(\sigma_{1} x_{1}=\alpha_{1}\right)\left(\sigma_{2} x_{2}=\right.\right.$ $\left.\left.\alpha_{2}\right) \ldots\left(\sigma_{n} x_{n}=\alpha_{n}\right)\right)$ be a disjunctive/conjunctive boolean equation system. The dependency graph of $\mathcal{E}$ is a triple $G_{\mathcal{E}}=(V, E, L)$ where

$-V=\{i \mid 1 \leq i \leq n\} \cup\{\perp, \top\}$ is the set of nodes

- $E \subseteq V \times V$ is the set of edges such that for all equations $\sigma_{i} x_{i}=\alpha_{i}$

- $(i, j) \in E$, if a variable $x_{j} \in \alpha_{i}$

- $(i, \perp) \in E$, if false occurs in $\alpha_{i}$

- $(i, \top) \in E$, if true occurs in $\alpha_{i}$

- $(\perp, \perp),(\top, \top) \in E$

$-L: V \rightarrow\{\mu, \nu\}$ is the node labeling defined by $L(i)=\sigma_{i}$ for $1 \leq i \leq n$, $L(\perp)=\mu$, and $L(\top)=\nu$.

Observe that in the definition above the sink nodes with self-loops, $\perp$ and $\top$, represent the constants false and true. The ordering on nodes (given by their sequence number) is extended to $\perp$ and $T$ by putting them highest in the ordering.

The key idea of our technique is based on the following observation that to obtain local solutions of variables in disjunctive/conjunctive equation systems, it suffices to compute the existence of a cycle in the dependency graph with certain properties.

Lemma 3. Let $G_{\mathcal{E}}=(V, E, L)$ be the dependency graph of a disjunctive (respectively conjunctive) boolean equation system $\mathcal{E}$. Let $x_{i}$ be any variable in $\mathcal{E}$ and let valuation $v$ be the solution of $\mathcal{E}$. Then the following are equivalent:

1. $v\left(x_{i}\right)=1$ (respectively $v\left(x_{i}\right)=0$ )

2. $\exists j \in V$ with $L(j)=\nu$ (respectively $L(j)=\mu$ ) such that:

a) $j$ is reachable from $i$, and

b) $G_{\mathcal{E}}$ contains a cycle of which the lowest index of a node on this cycle is $j$.

Proof. We only prove this lemma for disjunctive boolean equation systems. The case for conjunctive equation systems is dual and goes in the same way. First we show that (2) implies (1). If $j$ lies on a cycle with all nodes with numbers larger 
than $j$, there are two possibilities. Either $j$ equals $\top$ or $1 \leq j \leq n$. In the last case, there is a sub-equation system of $\mathcal{E}$ that looks as follows:

$$
\begin{gathered}
\nu x_{j}=\alpha_{j} \\
\vdots \\
\sigma_{k_{1}} y_{k_{1}}=\alpha_{k_{1}} \\
\sigma_{k_{2}} y_{k_{2}}=\alpha_{k_{2}} \\
\vdots \\
\sigma_{k_{n}} y_{k_{n}}=\alpha_{k_{n}}
\end{gathered}
$$

where $x_{j} \in \alpha_{j}\left[y_{k_{1}}:=\alpha_{k_{1}}\right]\left[y_{k_{2}}:=\alpha_{k_{2}}\right]\left[y_{k_{3}}:=\alpha_{k_{3}}\right] \ldots\left[y_{n}:=\alpha_{k_{n}}\right]$. Using lemma 2 we can rewrite the boolean equation system $\mathcal{E}$ to an equivalent one by replacing the equation $\nu x_{j}=\alpha_{j}$ by:

$$
\nu x_{j}=\alpha_{j}\left[y_{k_{1}}:=\alpha_{k_{1}}\right]\left[y_{k_{2}}:=\alpha_{k_{2}}\right]\left[y_{k_{3}}:=\alpha_{k_{3}}\right] \ldots\left[y_{n}:=\alpha_{k_{n}}\right] .
$$

Now note that the right hand side contains only disjunctions and the variable $x_{j}$ at least once. Hence, by lemma 2 the equation reduces to

$$
\nu x_{j}=\text { true }
$$

Now, as $x_{j}$ is reachable from $x_{i}$, the equation $\sigma_{i} x_{i}=\alpha_{i}$ can similarly be replaced by $\sigma_{i} x_{i}=$ true. Hence, for any solution $v$ of $\mathcal{E}$, it holds that $v\left(x_{i}\right)=1$. In case $j$ equals $T$, the term true is reachable from $x_{i}$. In a similar way using lemma 2 we can replace $\sigma_{i} x_{i}=\alpha_{i}$ by $\sigma_{i} x_{i}=$ true.

Now we prove that (1) implies (2) by contraposition. So, assume that there is no $j$ with $L(j)=v$ that is reachable from $i$ such that $j$ is on a cycle with only higher numbered nodes.

We prove with induction on $n-k$ that $\mathcal{E}$ is equivalent to the same boolean equation system where equations $\sigma_{k+1} x_{k+1}=\alpha_{k+1}, \ldots, \sigma_{n} x_{n}=\alpha_{n}$ that are reachable from $x_{i}$, have been replaced by $\sigma_{k+1} x_{k+1}=\beta_{k+1}, \ldots, \sigma_{n} x_{n}=\beta_{n}$ where all $\beta_{l}$ are disjunctions of false and variables that stem from $x_{1}, \ldots, x_{k}$. If the inductive proof is finished, the lemma is also proven: consider the case where $n-k=n$. This says that $\mathcal{E}$ is equivalent to a boolean equation system where all right hand sides of equations reachable from $x_{i}$ are equal to false. So, in particular $x_{i}=$ false, or in other words, for every solution $v$ of $\mathcal{E}$ it holds that $v\left(x_{i}\right)=0$.

For $n-k=0$ the induction hypothesis obviously holds. In particular true cannot occur in the right hand side of any equation reachable from $x_{i}$. So, consider some $n-k$ for which the induction hypothesis holds. We show that it also holds for $n-k+1$. So, we must show if equation $\sigma_{k} x_{k}=\alpha_{k}$ is reachable from $x_{i}$, it can be replaced by an equation $\sigma_{k} x_{k}=\beta_{k}$ where in $\beta_{k}$ only variables chosen from $x_{1}, \ldots, x_{k-1}$ and false can occur.

As $x_{k}$ is reachable from $x_{i}$, all variables $x_{l}$ occuring in $\alpha_{k}$ are also reachable from $x_{i}$. By the induction hypothesis the equations $\sigma_{l} x_{l}=\alpha_{l}$ for $l>k$ have been replaced by $\sigma_{l} x_{l}=\beta_{l}$ where in $\beta_{l}$ only false and variables from $x_{1}, \ldots, x_{k}$ 
occur. Using lemma 2 such variables $x_{l}$ can be replaced by $\beta_{l}$ and hence, $\alpha_{k}$ is replaced by $\gamma_{k}$ in which false and variables from $x_{1}, \ldots, x_{k}$ can occur.

What remains to be done is to remove $x_{k}$ from $\gamma_{k}$ assuming $x_{k} \in \gamma_{k}$. This can be done as follows. Suppose $\sigma_{k}$ equals $\nu$, then, as $x_{k}$ occurs in $\gamma_{k}$, there must be a path in the dependency graph to a node $x_{l^{\prime}}$ with $l^{\prime}>k$ such that $x_{k} \in \alpha_{l^{\prime}}$. But this means that the dependency graph has a cycle on which $k$ is the lowest value. This contradicts the assumption. So, it cannot be that $\sigma_{k}=\nu$, so, $\sigma_{k}=\mu$. Now using lemma 1 the variable $x_{k}$ in $\alpha_{k}$ can be replaced by false and subsequently be eliminated. This finalizes the induction step of the proof.

Now consider a disjunctive/conjunctive straight boolean equation system $\mathcal{E}$. In order to find a solution for $\mathcal{E}$ we first partition the set of variables $\mathcal{X}$ into blocks such that variables are in the same block iff these are mutually dependent. As $\mathcal{E}$ is disjunctive/conjunctive straight, all variables in each block have defining equations that are either disjunctive or conjunctive. Using the well known algorithm [15] for the detection of strongly connected components, the partition can be constructed in linear time on the basis of the variable dependency graph. As argued earlier, the equations belonging to the variables in each block can be solved iteratively. If the variables in a block do not depend on unsolved variables, the equations in this block can be solved. So, we only have to concentrate on solving disjunctive or conjunctive equations belonging to variables in a single block.

So, we present here an algorithm to solve a disjunctive boolean equation system. The conjunctive case is dual and goes along exactly the same lines. Our algorithm is an extension of Tarjan's [15] algorithm to detect strongly connected components. It is given in figure 1 and explained below.

We assume that the boolean equation system has already been transformed into a variable dependency graph $G=(V, E, L)$. There are two main functions solve and find. The function solve takes the index $i$ of a variable $x_{i}$ of interest and solves it by reporting it to be either 0 or 1 . The procedure find $(k)$ constructs all the strongly connected components from node $k$ and applies lemma 3 to them.

We use a standard adjacency-list representation and keep an array of lists of nodes. We assume that an array sign is given that indicates the label for each node. I.e. $\operatorname{sign}[i]=\nu$ if the label of node $i$ is $L(i)=\nu$, and $\operatorname{sign}[i]=\mu$ if the label of node $i$ is $L(i)=\mu$.

We keep an integer array value, initially set to all zeros, containing numbers indicating the order in which nodes have been visited. If value $[i]=0$, this indicates that node $i$ has not yet been visited. In addition, we keep a stack of integers, stack, represented as an array of size $|V|$ with a stack pointer $p$ initially set to zero. We have integers id (initially zero), min, and $m$ for the detection of SCCs, which occur in a similar vein in the algorithm for the detection of SCCs in [15]. The variable id is used to number the nodes with consecutive numbers in the sequence they are visited by the algorithm. The variable min refers to an earlier visited node, reachable from node $k$. If no such node exists, $\min =$ value $[k]$ at the end of the first for loop and node $k$ is the root of a strongly connected component that includes all higher numbered nodes residing on the stack. The 

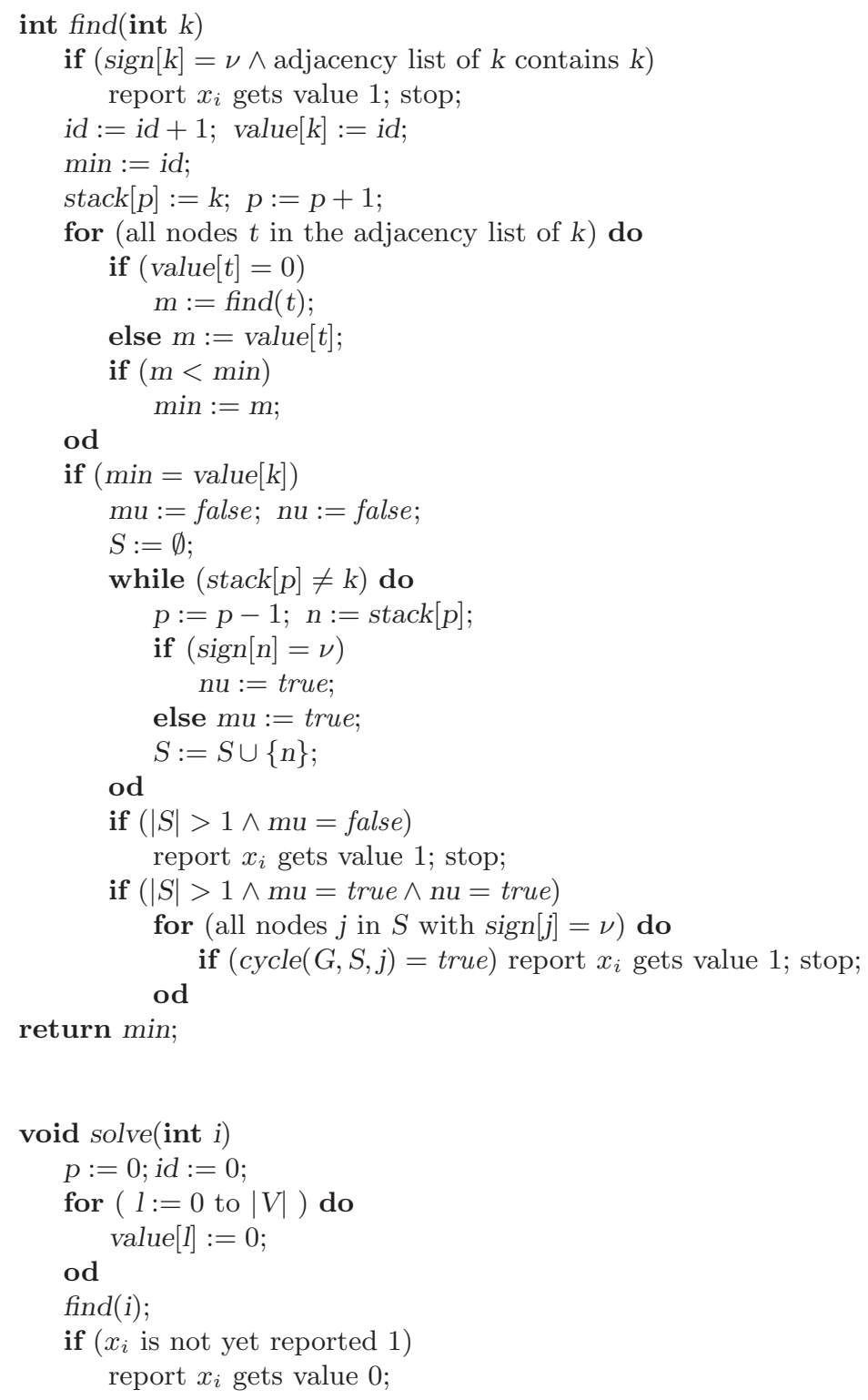

Fig. 1. An algorithm for alternating, disjunctive boolean equation systems. 
variable $m$ plays the role of a simple auxiliary store. Finally, we keep also a set $S$, integer $n$, and booleans $m u$ and $n u$ for processing the SCCs, explained below.

The procedure solve invokes the recursive procedure find. The procedure find first checks whether the node $k$ being visited is labelled with $\nu$ and has a self-loop. If these hold, we have found a node that trivially satisfies conditions (2a) and (2b) of lemma 3 , and the solution $v\left(x_{i}\right)=1$ can be reported and the execution of the algorithm is terminated. Otherwise, find pushes the nodes onto a stack, and recursively searches for strongly connected components. If such a component is found (when $\min =$ value $[k]$ ), find puts all nodes in the component that reside on the stack in a set $S$. While doing so, it is checked whether all nodes in the component have the same label. If a label is $\nu$, corresponding to the fixed point operator $\nu$, the variable $n u$ is set to true, and if a label is $\mu$, corresponding to polarity $\mu$, the variable $m u$ is set to true. If $m u=$ false on a SCC with more than one node, all nodes have label $\nu$ and so, conditions (2a) and (2b) of lemma 3 are trivially satisfied, and solution of $x_{i}$ can be reported to 1 .

If both variables $n u$ and $m u$ are true, the component is alternating. In this case it must be checked whether the SCC contains a cycle of which the smallest numbered node $j$ has label $L(j)=\nu$, according to lemma 3 to justify $x_{i}$ to be set to 1 . This is simply checked by applying a procedure $\operatorname{cycle}\left(G_{\mathcal{E}}, S, j\right)$ to all nodes $j \in S$ with $\operatorname{sign}[j]=\nu$. The procedure cycle consists of a simple linear depth first search and is not given in detail here.

Finally, if no node $j$ with $L(j)=\nu$ satisfying conditions $(2 \mathrm{a})$ and $(2 \mathrm{~b})$ of lemma 3 was found, we can report at the end of the procedure solve the solution $v$ of $\mathcal{E}$ be such that $v\left(x_{i}\right)=0$.

We find that the algorithm is correct and works in polynomial time and space.

Theorem 1. The algorithm for local resolution works correctly on any purely disjunctive/conjunctive system of boolean equations.

In order to formally estimate the computational costs, denote the set of alternating variables in a system $\mathcal{E}$ with variables in $\mathcal{X}$ by $\operatorname{alt}(\mathcal{E})$, and define it as a set $\left\{x_{i} \mid x_{i} \in \mathcal{X}\right.$ and $x_{i}$ is mutually dependent with some $x_{j} \in \mathcal{X}$ such that $\left.\sigma_{i} \neq \sigma_{j}\right\}$. The set of alternation free variables is denoted by $a f(\mathcal{E})$ and is defined as $a f(\mathcal{E})=\mathcal{X}-\operatorname{alt}(\mathcal{E})$. Note that for alternation-free boolean equation systems it holds that $\operatorname{alt}(\mathcal{E})=\emptyset$, because there are no ocurrences of mutually dependent variables with different signs. Then, it is easy to see that:

Theorem 2. The algorithm for local resolution of disjunctive/conjunctive boolean equation systems requires time $O\left(\right.$ af $(\mathcal{E})+$ alt $\left.(\mathcal{E})^{2}\right)$ and space $O(|\mathcal{E}|)$.

\section{Some Experiments}

In this section, we describe an implementation of the resolution algorithm presented in the previous section. This prototype solver for alternating disjunctive/conjunctive boolean equation systems is implemented in $\mathrm{C}$. To give an 
impression of the performance, we report experimental results on solving two verification problems using the tool.

As benchmarks we used two sets of $\mu$-calculus model checking problems taken from [11 and 14, converted to boolean equation systems. We do not take exactly the same formulas because our algorithm solves these in constant time, which would not give interesting results. The verification problems consist of checking $\mu$-calculus formulas of alternation depth 2 , on a sequence of regular labelled transition systems $M_{k}$ of increasing size (see figure 2).

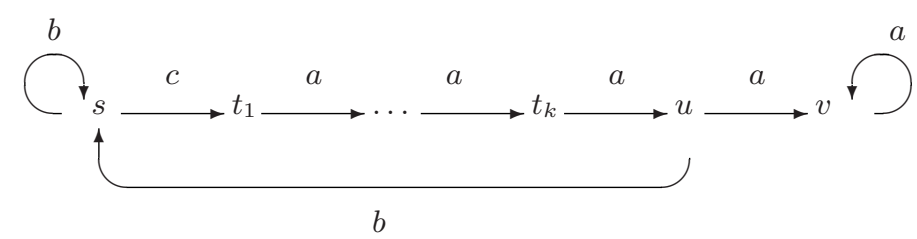

Fig. 2. Process $M_{k}$ for model checking the properties $\phi_{1}$ and $\phi_{2}$.

Suppose we want to check, at initial state $s$ of process $M_{k}$, the property that transitions labeled $b$ occur infinitely often along every infinite path of the process. This is expressed with alternating fixed-point formula:

$$
\phi_{1} \equiv \nu X . \mu Y \cdot([b] X \wedge[-b] Y)
$$

The property is false at state $s$ and we use the solver to find a counter-example for the formula. In second series of examples, we check the property that there is an execution in $M_{k}$ starting from state $s$, where action $a$ occurs infinitely often. This is expressed with the alternating fixed point formula

$$
\phi_{2} \equiv \nu X . \mu Y .(\langle a\rangle X \vee\langle-a\rangle Y)
$$

which is true at initial state $s$ of the process $M_{k}$.

The problems of determining whether the system $M_{k}$ satisfies the specifications $\phi_{1}$ and $\phi_{2}$ can be directly encoded as problems of solving the corresponding alternating boolean equation systems, which are in conjunctive and disjunctive forms. We report the times for the solver to find the local solutions corresponding to the local model checking problems of the formulas at state $s$.

The experimental results are given in table 1. The columns are explained below:

- Problem:

- the process $M_{k}$, with $k+3$ states

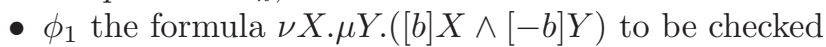

- $\phi_{2}$ the formula $\nu X . \mu Y .(\langle a\rangle X \vee\langle\neg a\rangle Y)$ to be checked 
Table 1. Summary of execution times.

\begin{tabular}{|c|c|c|c|}
\hline \multicolumn{2}{|c|}{ Problem } & $\mathrm{n}$ & Time $(\mathrm{sec})$ \\
\hline$M_{5000000}$ & $\phi_{1}$ & 10000006 & 2.6 \\
\cline { 2 - 4 } & $\phi_{2}$ & 10000006 & 3.0 \\
\hline$M_{10000000}$ & $\phi_{1}$ & 20000006 & 5.5 \\
\cline { 2 - 4 } & $\phi_{2}$ & 20000006 & 6.4 \\
\hline$M_{15000000}$ & $\phi_{1}$ & 30000006 & 7.5 \\
\cline { 2 - 4 } & $\phi_{2}$ & 30000006 & 9.0 \\
\hline
\end{tabular}

- $n$ : the number of equations in the boolean equation system corresponding to the model checking problem

- Time: the time in seconds to find the local solution

The times reported are the time for the solver to find the local solutions measured as system time, on a $2.4 \mathrm{Ghz}$ Intel Xeon running linux (i.e. the times for the solver to read the equation systems from disk and build the internal data structure are excluded).

In the problem with the property $\phi_{1}$, the solver found local solutions (and counterexamples) even without executing the quadratic part of the algorithm. In the problem with property $\phi_{2}$, the quadratic computation needed to be performed only on very small portions of the equation systems. These facts are reflected in the performance of the solver, which exhibits linear growth in the execution times with increase in the size of the systems to be verified, in all of the experiments.

The benchmarks in [1] and [14] are essentially the only benchmarks in the literature for alternating boolean equation systems of which we are aware. These benchmarks have a quite simple structure, and therefore we must be careful in drawing general results from them. A more involved practical evaluation is desireable here and benchmarking on real world protocols and systems is left for future work.

\section{Discussion and Conclusion}

We argued that the verification of many formulas in the modal mu-calculus with alternating fixed points amounts to the verification of disjunctive/conjunctive straight boolean equation systems. Subsequently we provided an algorithm to solve these and showed that the performance of this algorithm on the standard benchmarks from the literature yield an improvement of many orders of magnitude. We believe that this makes the verification of a large class of formulas with alternating fixed points tractable, even for large, practical systems.

The algorithm that we obtain is for the large part linear, but contains an unpleasant quadratic factor. Despite several efforts, we have not been able to eliminate this. In essence this is due to the fact that we were not able to find a sub-quadratic algorithm for the following problem: 
Open problem. Given a directed labelled graph $G=(V, E, L)$ of which the set of nodes is totally ordered. The labeling $L: V \rightarrow\{0,1\}$ assigns to each node a value. Determine whether there exist a cycle in $G$ of which the highest node has label 1.

As we believe that this problem has some interest by itself we provide it here.

Acknowledgements. We thank Michel Reniers for commenting a draft of this paper. The work of second author was supported by Academy of Finland (project 53695), Emil Aaltonen foundation and Helsinki Graduate School in Computer Science and Engineering.

\section{References}

1. H.R. Andersen. Model checking and boolean graphs. Theoretical Computer Science, 126:3-30, 1994.

2. A. Arnold and P. Crubille. A linear time algorithm to solve fixed-point equations on transition systems. Information Processing Letters, 29:57-66, 1988.

3. A. Arnold and D. Niwinski. Rudiments of $\mu$-calculus. Studies in Logic and the foundations of mathematics. Volume 146, Elsevier, 2001.

4. J. Bradfield and C. Stirling. Modal Logics and mu-Calculi: An introduction. Chapter 4 of Handbook of Process Algebra. J.A. Bergstra, A. Ponse and S.A. Smolka, editors. Elsevier, 2001.

5. G. Bhat and R. Cleaveland. Efficient local model-checking for fragments of the modal $\mu$-calculus. In Proceedings of the Int. Conf. on Tools and Algorithms for the Construction and Analysis of Systems, Lecture Notes in Computer Science 1055, pages 107-126, Springer Verlag 1996.

6. R. Cleaveland and B. Steffen. Computing Behavioural relations logically. In proceedings of the 18 International Colloquium on Automata, Languages and Programming, Lecture Notes Computer Science 510, pages 127-138, Springer Verlag, 1991.

7. E.A. Emerson, C. Jutla and A.P. Sistla. On model checking for fragments of the $\mu$-calculus. In C. Courcoubetis, editor, Fifth Internat. Conf. on Computer Aided Verification, Elounda, Greece, Lecture Notes in Computer Science 697, pages 385396, Springer Verlag, 1993.

8. E.A. Emerson, C. Jutla, and A.P. Sistla. On model checking for the $\mu$-calculus and its fragments. Theoretical Computer Science 258:491-522, 2001.

9. M. Jurdzinski. Deciding the winner in parity games is in $U P \cap c o-U P$. Information Processing Letters, 68:119-124, 1998.

10. D. Kozen. Results on the propositional $\mu$-calculus. Theoretical computer Science 27:333-354, 1983.

11. X. Liu, X, C.R. Ramakrishnan and S.A. Smolka. Fully Local and Efficient Evaluation of Alternating Fixed Points. In B. Steffen, editor, Proceedings of TACAS'98, Lecture Notes in Computer Science 1384, Springer Verlag, 1988.

12. A. Mader. Verification of Modal Properties using Boolean Equation Systems. PhD thesis, Technical University of Munich, 1997. 
13. R. Mateescu. A Generic On-the-Fly Solver for Alternation-Free Boolean Equation Systems. Proceedings of the 9th International Conference on Tools and Algorithms for the Construction and Analysis of Systems TACAS'2003 (Warsaw, Poland), volume 2619 of Lecture Notes in Computer Science, pages 81-96. Springer Verlag, April 2003.

14. B. Steffen, A. Classen, M. Klein, J. Knoop and T. Margaria. The fixpoint analysis machine. In I. Lee and S.A. Smolka, editors, Proceedings of the Sixth International Conference on Concurrency Theory (CONCUR '95), Lecture Notes in Computer Science 962, pages 72-87. Springer Verlag, 1995.

15. R. Tarjan. Depth-First Search and Linear Graph Algorithms. SIAM J. Computing, Vol. 1, No. 2, June 1972.

16. B. Vergauwen and J. Lewi. A linear algorithm for solving fixed-point equations on transition systems. In J.-C. Raoult, editor, CAAP'92, Lecture Notes Computer Science 581, pages 321-341, Springer Verlag, 1992.

17. B. Vergauwen and J. Lewi. Efficient Local Correctness Checking for Single and Alternating Boolean Equation Systems. In proc. of ICALP'94. 